\title{
A New Extended Burr XII Distribution
}

Indranil Ghosh*

University of North Carolina Wilmington Universidade do Rio Grande do Norte

Marcelo Bourguignon

\begin{abstract}
In this paper, we propose a new lifetime distribution, namely the extended Burr XII distribution (using the technique as mentioned in Cordeiro et al. (2015)). We derive some basic properties of the new distribution and provide a Monte Carlo simulation study to evaluate the maximum likelihood estimates of model parameters. For illustrative purposes, two real life data sets have been considered as an application of the proposed model.
\end{abstract}

Keywords: Burr XII distribution, estimation, half logistic family of distributions.

\section{Introduction}

The armory of statistical distributions is truly illimitable. New distributions are being unearthed literally on a weekly basis elicited by either theoretical considerations or by pressing practical applications or both. However, in various spheres of applied areas, for example, survival data analysis, finance and risk modeling, insurance, modeling rare events and biology, it has become imperative to develop an extended class of classical distributions to enhance its flexibility in modeling real life data that appears to have high degree of skewness and kurtosis. In the last few years, new classes of distributions were defined by extending the Weibull distribution to cope with bathtub shaped failure rates. Mudholkar and Srivastava (1993) and Mudholkar et al. (1996) pioneered and studied the exponentiated Weibull distribution to analyze bathtub failure data. A good review of some of these extended models is presented in Pham and Lai (2007).

In this paper, we consider the technique of mixing two absolutely continuous distributions. We consider here the particular case of the general Type I half logistic family of distributions, studied by Cordeiro et al. (2015). The cumulative distribution function (cdf) and the probability density function (pdf) is given by

$$
F(x ; \lambda, \xi)=\int_{0}^{-\log [1-G(x ; \xi)]} \frac{2 \lambda \mathrm{e}^{-\lambda t}}{\left(1+\mathrm{e}^{-\lambda t}\right)^{2}} d t=\frac{1-[1-G(x ; \xi)]^{\lambda}}{1+[1-G(x ; \xi)]^{\lambda}},
$$

where $G(x ; \xi)$ is the baseline cdf depending on a parameter vector $\xi$ and $\lambda>0$ is an additional shape parameter. Also, the corresponding pdf will be

$$
f(x ; \lambda, \xi)=\frac{2 \lambda g(x ; \xi)[1-G(x ; \xi)]^{\lambda-1}}{\left\{1+[1-G(x ; \xi)]^{\lambda}\right\}^{2}} .
$$


Next, if we consider the baseline distribution as $G(x)=1-\left(1+x^{c}\right)^{-1}$ (Burr XII distribution), then, the pdf and the cdf for a extended Burr XII distribution with parameters $c$ and $\lambda$ are respectively,

$$
f(x ; \lambda, c)=\frac{2 c \lambda x^{c-1}\left(1+x^{c}\right)^{\lambda-1}}{\left[1+\left(1+x^{c}\right)^{\lambda}\right]^{2}}, \quad x>0, \quad c>0,
$$

and

$$
F(x ; \lambda, c)=1-\frac{2}{1+\left(1+x^{c}\right)^{\lambda}} .
$$

If a random variable $X$ has the density as in (1), then we say that $X$ follows Extended Burr XII (henceforth EBXII in short) distribution with parameters $c$ and $\lambda$. One significant usefulness of Equation (1) is its ability to analyze skewed data that can not be properly fitted by its parent distributions. Furthermore, it permits greater flexibility in its tails and can be widely applied in many areas of reliability and biology. The proposed distribution has only two parameters and the density and the cumulative distribution functions of the new distribution are simple, i.e., the EBXII distribution is in fact very tractable. We mention here an important fact that our derived model, although bears the same name as in Shao et al. (2004) and Mudholkar et al. (1996), who alternatively called this distribution as generalized Weibull, is completely different from all of them in terms of the genesis of the model and subsequently its stochastic properties.

The rest of the paper is organized as follows. In Section 2, we discuss briefly some properties of the extended Burr XII (henceforth EBXII) distribution. These include reliability parameter and order statistics. In Section 3, we discuss the likelihood inference for the EBXII and conduct a simulation study for specific choices of the model parameters. In Section 4, we illustrate the applicability of the EBXII with two data sets and compared with the rival Burr XII distribution. Finally, in Section 5, we provide some concluding remarks.

\section{Properties of the extended Burr XII distribution}

In this section, we consider some properties of the EBXII distribution, such as the reliability parameter and order statistics. Hereafter, the random variable $X$ following Equation (2) with parameters $c$ and $\lambda$ is denoted by $X \sim \operatorname{EBXII}(c, \lambda)$.

The reliability parameter $R$ is defined as $R=P(X>Y)$, where $X$ and $Y$ are independent random variables. If $X$ and $Y$ are two continuous and independent random variables with the cdfs $F_{1}(x)$ and $F_{2}(y)$ and their pdfs $f_{1}(x)$ and $f_{2}(y)$ respectively. Then the reliability parameter $R$ can be written as

$$
R=P(X>Y)=\int_{-\infty}^{\infty} F_{2}(t) f_{1}(t) d t
$$

Theorem 1. Suppose that $X \sim \operatorname{EBXII}\left(c, \lambda_{1}\right)$ and $Y \sim \operatorname{EBXII}\left(c, \lambda_{2}\right)$, and they are independent. Then

$$
P(X>Y)=1-4 B\left(\frac{\lambda_{2}}{\lambda_{1}}+1,1-\frac{\lambda_{2}}{\lambda_{1}}\right) .
$$

Proof: From (1) and (2), we have

$$
\begin{aligned}
P(X>Y) & =\int_{0}^{\infty}\left[1-\frac{2}{1+\left(1+t^{c}\right)^{\lambda_{2}}}\right]\left\{\frac{2 c \lambda t^{c-1}\left(1+t^{c}\right)^{\lambda_{1}-1}}{\left[1+\left(1+t^{c}\right)^{\lambda_{1}}\right]^{2}}\right\} d t \\
& =1-4 \int_{1}^{\infty} u^{-2}(u-1)^{\frac{\lambda_{2}}{\lambda_{1}}} d u, \quad \text { on substitution } u=1+\left(1+t^{c}\right)^{\lambda_{1}} \\
& =1-4 B\left(1+\frac{\lambda_{2}}{\lambda_{1}}, 1-\frac{\lambda_{2}}{\lambda_{1}}\right) .
\end{aligned}
$$


Now, we will consider the expression for the general $r$-th order statistic and the large sample distribution of the sample minimum and the sample maximum when a random sample of size $n$ are drawn from the EBXII distribution. The density function of the $r$-th order statistic, $X_{r: n}$, for a random sample of size $n$ drawn from (1) is given by

$$
f_{X_{r: n}}(x)=\frac{f(x)}{B(r, n-r+1)} \sum_{j=0}^{r-1}(-1)^{j}\left(\begin{array}{c}
r-1 \\
j
\end{array}\right)[1-F(x)]^{n-r+j} I(0<x<\infty) .
$$

Thus the pdf of $X_{r: n}$ can be alternatively written as

$$
f_{r: n}(x)=\frac{1}{B(r, n-r+1)} \sum_{j=0}^{r-1}(-1)^{j}\left(\begin{array}{c}
r-1 \\
j
\end{array}\right) \frac{2^{n-r+j+1} c \lambda x^{c-1}\left(1+x^{c}\right)^{\lambda-1}}{\left[1+\left(1+x^{c}\right)^{\lambda}\right]^{2+n-r+j}} I(0<x<\infty) .
$$

In order to derive the asymptotic distribution of the sample minima $X_{1: n}$, we consider Theorem 8.3.6 of Arnold et al. (2008). Observe that, since $F^{-1}(0)=0$, it follows from the theorem that the asymptotic distribution of the sample minima $X_{1: n}$ is not of Fréchet type. The asymptotic distribution of $X_{1: n}$ will be of Weibull type with parameter $\delta>0$ if

$$
\lim _{\varepsilon \rightarrow 0_{+}} \frac{F(\varepsilon x)}{F(\varepsilon)}=x^{\delta}, \quad \text { for all } \quad x>0 .
$$

By using L'Hôpital's rule, it follows that

$$
\lim _{\varepsilon \rightarrow 0_{+}} \frac{F(\varepsilon x)}{F(\varepsilon)}=x \lim _{\varepsilon \rightarrow 0_{+}} \frac{f(\varepsilon x)}{f(\varepsilon)}=x \lim _{\varepsilon \rightarrow 0_{+}} \frac{\frac{2 c \lambda(\varepsilon x)^{c-1}\left(1+(\varepsilon x)^{c}\right)^{\lambda-1}}{\left(1+\left(1+(\varepsilon x)^{c}\right)^{\lambda}\right)^{2}}}{\frac{2 c \lambda \varepsilon^{c-1}\left(1+x^{c}\right)^{\lambda-1}}{\left(1+\left(1+\varepsilon^{c}\right)^{\lambda}\right)^{2}}}=x^{c} \lim _{\varepsilon \rightarrow 0_{+}} \frac{\frac{\left(1+(\varepsilon x)^{c}\right)^{\lambda-1}}{\left(1+\left(1+(\varepsilon x)^{c}\right)^{\lambda}\right)^{2}}}{\frac{\left(1+\varepsilon^{c}\right)^{\lambda-1}}{\left(1+\left(1+\varepsilon^{c}\right)^{\lambda}\right)^{2}}} .
$$

Hence,

$$
\lim _{\varepsilon \rightarrow 0_{+}} \frac{F(\varepsilon x)}{F(\varepsilon)}=x^{c} .
$$

Hence we obtain that the asymptotic distribution of the sample minima $X_{1: n}$ is of the Weibull type with shape parameter $c$. The asymptotic distribution of the sample maxima $X_{n: n}$, can be viewed as $F_{n}(x)$, where $F_{n}(x)=1-F_{1}(-x)$, where $F_{1}($.$) is the cdf of X_{1: n}$.

\section{Maximum likelihood estimation}

In this section, we address the parameter estimation of the $\operatorname{EBXII}(c, \lambda)$ under the classical set up. Let $x_{1}, x_{2}, \cdots, x_{n}$ be a random sample of size $n$ drawn from the density in (1). The log-likelihood function is given by

$$
\ell=n \log (2 c \lambda)+(c-1) \sum_{i=1}^{n} \log \left(x_{i}\right)+(\lambda-1) \sum_{i=1}^{n} \log \left(1+x_{i}^{c}\right)-2 \sum_{i=1}^{n} \log \left[1+\left(1+x_{i}^{c}\right)^{\lambda}\right] .
$$

The derivatives of (3) with respect to $c$ and $\lambda$ are given by

$$
\begin{gathered}
\frac{\partial}{\partial c} \ell=\frac{n}{c}+\sum_{i=1}^{n} \log \left(x_{i}\right)+\sum_{i=1}^{n} x_{i}^{c}\left(1+x_{i}^{c}\right)^{-1} \log \left(x_{i}\right)\left\{(\lambda-1)-2 \lambda\left(1+x_{i}^{c}\right)^{\lambda}\left[1+\left(1+x_{i}^{c}\right)^{\lambda}\right]^{-1}\right\} . \\
\frac{\partial}{\partial \lambda} \ell=\frac{n}{\lambda}+\sum_{i=1}^{n} \log \left(1+x_{i}^{c}\right)-2 \sum_{i=1}^{n}\left[1+\left(1+x_{i}^{c}\right)^{\lambda}\right]^{-1}\left(1+x_{i}^{c}\right)^{\lambda} \log \left(1+x_{i}^{c}\right) .
\end{gathered}
$$


The MLE $\hat{c}$ and $\hat{\lambda}$ are obtained by setting (4) and (5) to zero and solving them simultaneously. Next, a small Monte Carlo simulation experiment is conducted to evaluate the maximum likelihood estimation of the EBXII distribution parameters. We set the sample size at $n=$ 50,100, 200, 400 and 800. The Monte Carlo simulation experiments are performed using the $\mathrm{R}$ programming language; see http://www.r-project.org. All results were obtained from 10,000 Monte Carlo replications and the simulations were carried out using the $\mathrm{R}$ programming language; see http://www.r-project.org.

Table 1 reports the empirical mean and the mean squared error (in parentheses) of the corresponding estimator. From this table, note that, as the sample size increases, the empirical bias and mean squared error decrease in all the cases analyzed, as expected.

Table 1: Empirical means and mean squared errors (in parentheses).

\begin{tabular}{rrrr}
\hline$c$ & $\lambda$ & $\widehat{c}$ & $\widehat{\lambda}$ \\
\hline \multicolumn{3}{c}{$n=50$} \\
\hline 0.5 & 0.5 & $0.5495(0.0465)$ & $0.4911(0.0204)$ \\
1.5 & 0.5 & $1.6357(0.2732)$ & $0.4839(0.0448)$ \\
0.5 & 1.5 & $0.5179(0.0058)$ & $1.5051(0.0469)$ \\
1.5 & 1.5 & $1.5442(0.0583)$ & $1.5067(0.0557)$ \\
\hline \multicolumn{3}{c}{$n=100$} \\
\hline 0.5 & 0.5 & $0.5188(0.0082)$ & $0.4968(0.0096)$ \\
1.5 & 0.5 & $1.5609(0.0824)$ & $0.4937(0.0181)$ \\
0.5 & 1.5 & $0.5075(0.0026)$ & $1.5021(0.0234)$ \\
1.5 & 1.5 & $1.5210(0.0264)$ & $1.5017(0.0269)$ \\
\hline \multicolumn{3}{r}{$n=200$} \\
\hline 0.5 & 0.5 & $0.5096(0.0035)$ & $0.4985(0.0036)$ \\
1.5 & 0.5 & $1.5308(0.0359)$ & $0.4931(0.0173)$ \\
0.5 & 1.5 & $0.5041(0.0013)$ & $1.4999(0.0117)$ \\
1.5 & 1.5 & $1.5101(0.0107)$ & $1.5030(0.0110)$ \\
\hline \multicolumn{3}{r}{$n=400$} \\
\hline 0.5 & 0.5 & $0.5049(0.0016)$ & $0.4989(0.0018)$ \\
1.5 & 0.5 & $1.5145(0.0162)$ & $0.4976(0.0058)$ \\
0.5 & 1.5 & $0.5020(0.0006)$ & $1.5007(0.0054)$ \\
1.5 & 1.5 & $1.5066(0.0054)$ & $1.5001(0.0054)$ \\
\hline \multicolumn{3}{r}{$n=800$} \\
\hline 0.5 & 0.5 & $0.5023(0.0008)$ & $0.4995(0.0009)$ \\
1.5 & 0.5 & $1.5066(0.0076)$ & $0.4993(0.0023)$ \\
0.5 & 1.5 & $0.5010(0.0003)$ & $1.5003(0.0027)$ \\
1.5 & 1.5 & $1.5037(0.0027)$ & $1.4996(0.0027)$ \\
\hline & &
\end{tabular}

\section{Applications}

For illustrative purposes, we consider two data sets and compare with the Burr XII (BXII) distribution. For each data set, we estimate the unknown parameters of each distribution by the maximum-likelihood method (as discussed in Section 3) and all the computations were done using the subroutine NLMixed of the SAS software. We obtain the values of the Akaike information criterion (AIC), Bayesian information criterion (BIC) and consistent Akaike information criterion (CAIC). First, we describe the three data sets:

- Data set I: The first example consist of thirty successive values of March precipitation (in inches) in Minneapolis/St Paul (Hinkley 1977). The data are: 0.77, 1.74, 0.81, 1.2, 1.95, 1.2, $0.47,1.43,3.37,2.2,3,3.09,1.51,2.1,0.52,1.62,1.31,0.32,0.59,0.81,2.81,1.87,1.18,1.35$, $4.75,2.48,0.96,1.89,0.9,2.05$. 
- Data set II: In the second data set, we consider vinyl chloride data obtained from clean upgradient monitoring wells in $\mathrm{mg} / \mathrm{L}$; this data set was used for Bhaumik et al. (2009). The data are: $5.1,1.2,1.3,0.6,0.5,2.4,0.5,1.1,8.0,0.8,0.4,0.6,0.9,0.4,2.0,0.5,5.3,3.2,2.7$, $2.9,2.5,2.3,1.0,0.2,0.1,0.1,1.8,0.9,2.0,4.0,6.8,1.2,0.4,0.2$.

Tables 2 and 3 provides some descriptive statistics and the MLEs (with corresponding standard errors in parentheses) of these two data sets. Since the values of the AIC, BIC and CAIC are smaller for the EBXII distribution compared with those values of the BXII model, this new distribution seems to be a very competitive model for these data.

Table 2: Descriptive statistics for the two data sets.

\begin{tabular}{lcccccccc}
\hline & $n$ & Min. & $Q_{1}$ & $Q_{2}$ & Mean & $Q_{3}$ & Max. & Var. \\
\hline Set I & 30 & 0.320 & 0.915 & 1.470 & 1.675 & 2.088 & 4.750 & 1.0012 \\
Set II & 34 & 0.100 & 0.500 & 1.150 & 1.879 & 2.475 & 8.0 & 3.8126 \\
\hline
\end{tabular}

Table 3: MLEs of the model parameters with corresponding SE's (given in parentheses) for the two data sets and the corresponding AIC, CAIC and BIC statistics.

\begin{tabular}{l|ccc|ccc}
\hline Set I & $\widehat{c}$ & $\widehat{\lambda}$ & $\widehat{k}$ & AIC & BIC & CAIC \\
\hline EBXII & 2.8689 & 0.8811 & - & 82.4 & 85.2 & 82.8 \\
& $(0.5977)$ & $(0.1979)$ & $(-)$ & & & \\
\hline BXII & 3.2555 & - & 0.5770 & 84.5 & 87.3 & 85.0 \\
& $(0.6455)$ & $(-)$ & $(0.1371)$ & & & \\
\hline \hline Set II & $\widehat{c}$ & $\widehat{\lambda}$ & $\widehat{k}$ & AIC & BIC & CAIC \\
\hline EBXII & 1.3457 & 1.3837 & - & 115.4 & 118.5 & 115.8 \\
& $(0.2405)$ & $(0.2405)$ & $(-)$ & & & \\
\hline BXII & 1.5621 & - & 0.9305 & 116.2 & 119.2 & 116.5 \\
& $(0.2479)$ & $(-)$ & $(0.1791)$ & & & \\
\hline
\end{tabular}

Plots of the pdf of the EBXII and BXII fitted models to these data are displayed in Figure 1. They indicate that the EBXII distribution is superior to the BXII distribution in terms of model fitting. Based on these plots, we conclude that the EBXII distribution provides a better fit to these data than the BXII model.

\section{Concluding remarks}

There has been a growing interest among statisticians and applied researchers in developing flexible lifetime models for the betterment of modeling survival data. In this paper, we introduce a two parameter extended Burr XII distribution which is obtained by considering a Burr XII distribution as the baseline cdf in the Cordeiro et al. (2015) model. We study some of its statistical and mathematical properties. Parameter estimation is approached by maximum likelihood. The usefulness of the new distribution is illustrated in an analysis of two real data sets. We hope that the proposed extended model will invite wider applications in survival analysis.

\section{Acknowledgments}

The authors are grateful to the editor and the two referees for their helpful comments and suggestions which has significantly improved this paper. 

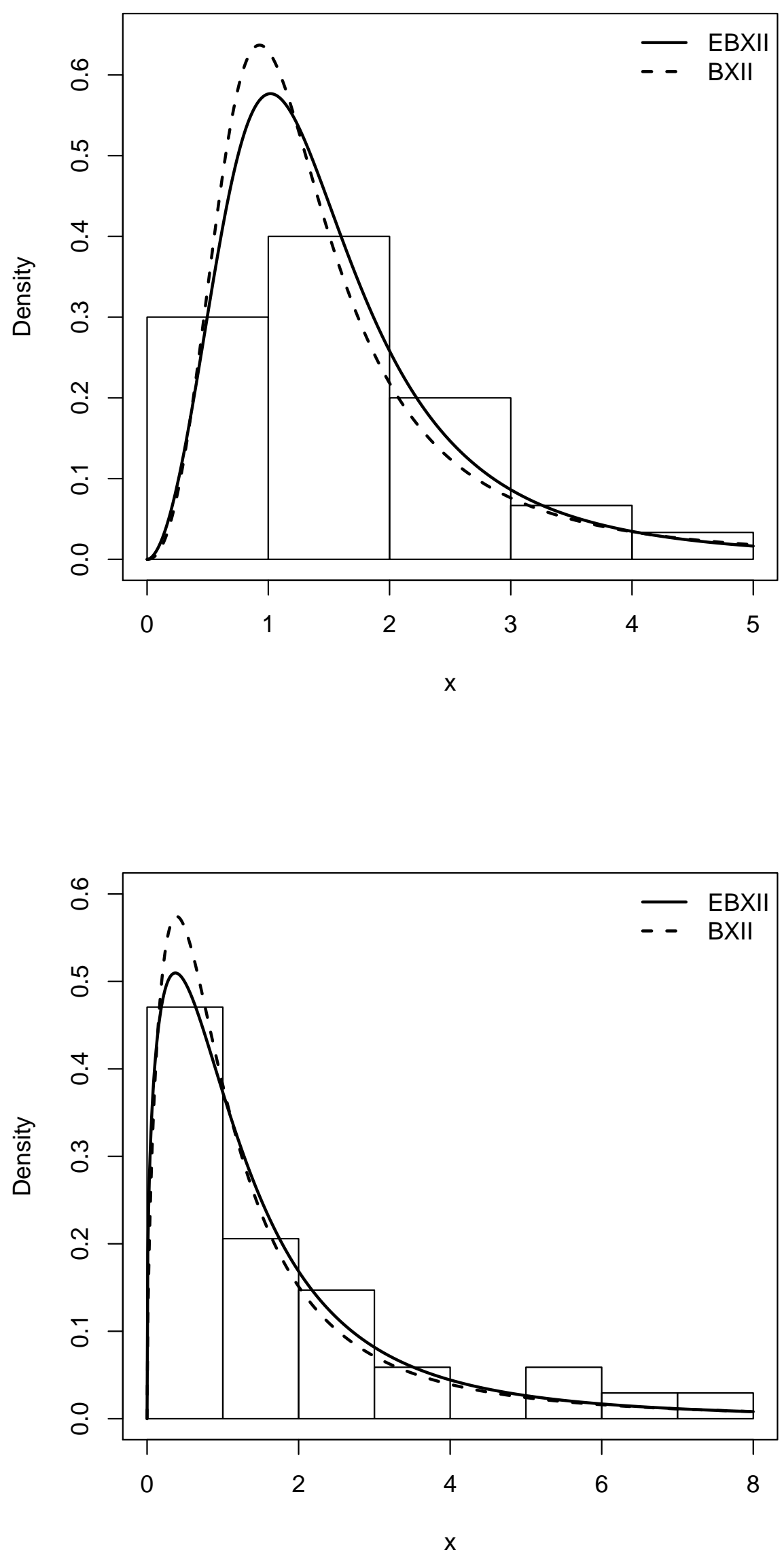

Figure 1: Plots of the fitted densities of the EBXII and BXII distributions for the first and second data sets. 


\section{References}

Arnold, B.C., Balakrishnan, N. and Nagaraja, H.N. (2008). A First Course in Order Statistics, Classic Edition. Society for Industrial and Applied Mathematics, Philadelphia, PA.

Cordeiro, G.M., Alizadeh, M. and Marinho, P.D. (2015). The Type I Half-Logistic Family of Distributions, Journal of Statistical Computation and Simulation, 86, 707-728.

Bhaumik, D.K., Kapur, K., Gibbons, R.D. (2009). Testing Parameters of a Gamma Distribution for Small Samples. Technometrics, 51, 326-334.

Hinkley, D. (1977). On Quick Choice of Power Transformation. Applied Statistics, 26, 67-69.

Mudholkar, G.S. and Srivastava, D.K. (1993). Exponentiated Weibull Family Analyzing Bathtub Failure-Rate Data. IEEE Transactions and Reliability, 42, 299-302.

Mudholkar, G.S., Srivastava, D. K. and Kollia, G.D. (1996). A Generalization of the Weibull Distribution With Application to the Analysis of Survival Data. Journal of the American Statistical Association, 91, 1575-1583.

Pham, H. and Lai, C.D. (2007). On Recent Generalizations of the Weibull Distribution. IEEE Transactions on Reliability, 56, 454-458.

Shao, Q.X., Wong, H., Xia, J. and I, W.C. (2004). Models for Extreme Using the Extended Three-Parameter Burr XII System With Application to Flood Frequency Analysis. Hydrological Sciences Journal, 49, 685-702.

\footnotetext{
Affiliation:

* Corresponding Author Indranil Ghosh

Department of Mathematics \& Statistics

University of North Carolina Wilmington

Wilmington, NC, USA

E-mail: ghoshi@uncw.edu
}

\section{Austrian Journal of Statistics}

published by the Austrian Society of Statistics

Volume 46

February 2017 http://www.ajs.or.at/

http://www.osg.or.at/

Submitted: 2015-10-20

Accepted: 2016-04-13 